\title{
A NOTE ON THE USE OF FROBENIUS MAP AND D-MODULES IN LOCAL COHOMOLOGY
}

\author{
MAJID EGHBALI
}

\begin{abstract}
The Frobenius depth denoted by F-depth defined by HartshorneSpeiser in 1977 and later by Lyubeznik in 2006, in a different way, for rings of positive characteristic. The first aim of the present paper is to compare the F-depth with formal grade and reprove some results of Lyubeznik using formal local cohomology. Then the endomorphism rings of local cohomology modules will be considered. As an application we reprove the results due to Huneke-Koh in positive characteristic and Lyubeznik in characteristic zero on the annihilators of local cohomology modules.
\end{abstract}

\section{INTRODUCTION}

Let $Y$ be a closed subscheme of $\mathbb{P}_{k}^{n}$, the projective space over a field $k$ of characteristic $p>0$. Vanishing of $H^{i}\left(\mathbb{P}^{n}-Y, \mathcal{F}\right)$ for all coherent sheaves $\mathcal{F}$ was asked by Grothendieck ([10]). Among the attempts to answer the mentioned question Hartshorne and Speicer in [12] used the notion of Frobenius depth of $Y$ to give an essentially complete solution to this problem.

To be more precise, Let $Y$ be a Noetherian scheme of finite dimension, whose local rings are all of characteristic $p>0$. Let $y \in Y$ be a (not necessarily closed) point. Let $d(y)$ be the dimension of the closure $\{y\}^{-}$of the point $y$. Let $\mathcal{O}_{y}$ be the local ring of $y$, let $k_{0}$ be its residue field, let $k$ be a perfect closure of $k_{0}$, and let $\widehat{\mathcal{O}}_{y}$, be the completion of $\mathcal{O}_{y}$. Choose a field of representatives for $k_{0}$ in $\widehat{\mathcal{O}}_{y}$. Then we can consider $\widehat{\mathcal{O}}_{y}$ as a $k_{0}$-algebra, and we let $A_{y}$ be the local ring $\widehat{\mathcal{O}}_{y} \otimes_{k_{0}} k$ obtained by base extension to $k$. Let $Y_{y}=\operatorname{Spec} A_{y}$ and let $P$ denote its closed point. So, the Frobenius depth of $Y$ denoted by F-depth $Y$ is the largest integer $r$ (or $+\infty$ ) such that for all points $y \in Y$, one has $H_{P}^{i}\left(Y_{y}, \mathcal{O}_{Y_{y}}\right)_{s}=0$ (the stable part of $\left.H_{P}^{i}\left(Y_{y}, \mathcal{O}_{Y_{y}}\right)\right)$ for all $i<r-d(y)$.

From the local algebra point of view, Grothendieck's problem is stated to find conditions under which $H_{I}^{i}(M)=0$ for all $i>n(n \in \mathbb{Z})$ and all $A$-modules $M$,

2000 Mathematics Subject Classification. 13D45, 14B15.

Key words and phrases. Frobenius depth, Positive characteristic methods, Local cohomology, formal grade, depth, D-modules, Annihilator.

This research was in part supported by a grant from IPM (No. 93130017). 
where $A$ is a commutative Noetherian local ring and $I \subset A$ is an ideal. For an $A$ module $M$, we denote by $H_{I}^{i}(M)$ the $i$ th local cohomology module of $M$ with respect to $I$. For more details the reader may consult $[9]$ and $[3]$. From the celebrated result of Hartshorne (cf. [11, pp. 413]), it is enough to find conditions for the vanishing of $H_{I}^{i}(A)$. In this direction, for a local ring $(A, \mathfrak{m})$, Lyubeznik in 21] using the Frobenius map from $H_{\mathfrak{m}}^{i}(A)$ to itself defined the Frobenius depth of $A$ denoted by F-depth $A$ as the smallest $i$ such that for every iteration of Frobenius map, $H_{\mathfrak{m}}^{i}(A)$ does not go to zero. It is noteworthy to say that the Lyubeznik's F-depth coincides with the notion of F-depth defined by Hartshorne and Speiser, whenever $A$ admits a surjection from a regular local ring and $Y=\operatorname{Spec} A$ (cf. [21, Corollary 6.3]).

Consider the family of local cohomology modules $\left\{H_{\mathfrak{m}}^{i}\left(M / I^{n} M\right)\right\}_{n \in \mathbb{N}}$ where, $(A, \mathfrak{m})$ is not necessarily of characteristic $p>0$. For every $n \in \mathbb{N}$ there is a natural homomorphism

$$
H_{\mathfrak{m}}^{i}\left(M / I^{n+1} M\right) \rightarrow H_{\mathfrak{m}}^{i}\left(M / I^{n} M\right)
$$

(induced from the natural projections $M / I^{n+1} M \rightarrow M / I^{n} M$ ) such that the family forms a projective system. The projective $\operatorname{limit}_{\lim _{n}} H_{\mathfrak{m}}^{i}\left(M / I^{n} M\right)$ is called the $i$ th formal local cohomology of $M$ with respect to $I$ (cf. [26]). Formal local cohomology modules were used by Peskine and Szpiro in 25] when $A$ is a regular ring of prime characteristic. It is noteworthy to mention that if $U=\operatorname{Spec}(A) \backslash\{\mathfrak{m}\}$ and $\left(\widehat{U}, \mathcal{O}_{\widehat{U}}\right)$ denotes the formal completion of $U$ along $V(I) \backslash\{\mathfrak{m}\}$ and For an $A$-module $M, \mathcal{F}$ denotes the associated sheaf on $U$ and $\widehat{\mathcal{F}}$ denote the coherent $\mathcal{O}_{\widehat{U}}$-sheaf associated to $\lim _{n} M / I^{n} M$, they have described the formal cohomology modules $H^{i}\left(\widehat{U}, \mathcal{O}_{\widehat{U}}\right)$ via the isomorphisms $H^{i}\left(\widehat{U}, \mathcal{O}_{\widehat{U}}\right) \cong \lim _{n} H_{\mathfrak{m}}^{i}\left(M / I^{n} M\right), i \geq 1$. See also 24, proposition (2.2)] when $A$ is a Gorenstein ring.

The formal grade, fgrade $(I, M)$, is defined as the index of the minimal nonvanishing formal cohomology module, i.e., fgrade $(I, M)=\inf \left\{i \in \mathbb{Z} \mid \lim _{n} H_{\mathfrak{m}}^{i}\left(M / I^{n} M\right) \neq\right.$ $0\}$. One way to check out vanishing of local cohomology modules is the following duality

$$
\lim _{\longleftarrow} H_{\mathfrak{m}}^{i}\left(A / I^{n}\right) \cong \operatorname{Hom}_{A}\left(H_{I}^{\operatorname{dim} A-i}(A), E(A / \mathfrak{m})\right)
$$

where $(A, \mathfrak{m})$ is a Gorenstein local ring and $E(A / \mathfrak{m})$ denotes the injective hull of the residue field (cf. [26, Remark 3.6]). To be more precise, in this case the last non vanishing amount of $H_{I}^{i}(A)$ may be described with the fgrade $(I, A)$. Thus, it motivates us to consider the invariants F-depth and fgrade to shed more light on the notion of Frobenius depth from a different point of view. For this reason, in Section 2, we bring some auxiliary results and among them we examine the structure of $\lim _{n} H_{\mathfrak{m}}^{i}\left(A / I^{n}\right)$ as a unite $A\left[F^{e}\right]$-module (Theorem 2.8). In Section 3, we show that the $F$-nilpotency of $H_{\mathfrak{m}}^{i}(A / I),(i \in \mathbb{Z})$ is equivalent to the vanishing 
of $\lim _{n} H_{\mathfrak{m}}^{i}\left(A / I^{n}\right)$ for a regular local $F$-finite ring $A$ (Proposition 3.1). It helps us to reprove 21, Corollary 3.2, Lemma 2.4], see Corollary 3.3 and Proposition 3.5. Finally, we compare the Frobenius depth with the formal grade and depth, (cf. Theorem 3.8 and Corollary 3.9).

Studying of the endomorphism rings of local cohomology modules are of interest in recent years. See for instance Hochster-Huneke [14, Hellus-Stückrad [13, Schenzel 27], 28], Eghbali-Schenzel 8], Mahmood-Schenzel [22]. In Section 4, we examine the endomorphism rings of local cohomology modules, $\operatorname{Hom}_{R}\left(H_{I}^{i}(R), H_{I}^{i}(R)\right)$, where $R$ is a regular local ring containing a field in both characteristic zero and $p>0$. Our way to prove the results is to use the so-called $\mathfrak{D}$-modules and Frobenius map. These methods have played decisive roles in many subsequent studies in the rings of characteristic zero and positive characteristic. As an application we reprove the results due to Huneke-Koh [16, Lemma 2.2] in positive characteristic and Lyubeznik [18, Corollary 3.6] in characteristic zero on the annihilators of local cohomology modules (cf. Corollaries 4.5 and 4.12).

\section{Auxiliary Results in POSITIVe Characteristic}

Throughout this section all rings are assumed to contain a field of positive characteristic. The symbol $A$ will always denote a commutative Noetherian ring of finite characteristic. We adapt the notation from [1] and except for notation we mostly follow Lyubeznik [19]. We let $F=F_{A}$ the Frobenius map on $A$, that is $F: A \rightarrow A$, with $a \mapsto a^{p}, a \in A$. We denote the eth iterate of the Frobenius map by $A^{e}$ which is the $A-A$-bimodule. As a left $A$-module it is $A$ and as a right $A$-module we have $m . a=a^{p^{e}} m$ for $m \in A^{e}$. We say $A$ is F-finite, whenever $A^{e}$ is a finitely generated right $A$-module.

Remark 2.1. Let us recall from [12, Proposition 1.1(a)] that for a ring $A$ which is either a localization of an algebra of finite type over a perfect field $k$, or a complete local ring containing a perfect field $k$ as its residue field, then $A$ is $F$-finite.

In the present section, among our results we recall various results due to HartshorneSpeiser [12], Peskine-Szpiro [25], Lyubeznik [19] and Blickle [1.

Peskine and Szpiro in [25] defined the Frobenius functor as follows:

Definition 2.2. The Frobenius functor is the right exact functor from $A$-modules to $A$-modules given by

$$
F_{A}^{*} M:=A^{1} \otimes_{A} M
$$

Its eth power is $F_{A}^{e *} M=A^{e} \otimes_{A} M$. For brevity we often write $F^{e *}$ for $F_{A}^{e *}$ when there is no ambiguity about the ring $A$. 
It follows from the definition that $F^{e *}$ commutes with direct sum, direct limit and localization. By a theorem of Kunz [17 the Frobenius functor is flat whenever $A$ is a regular ring, hence in this case $F^{e^{*}}$ will be exact and immediately one has $F^{e *} A=A^{e} \otimes_{A} A \cong A$ and $F^{e *} I=I^{\left[p^{e}\right]}$ an ideal of $A$ generated by $p^{e}$ th powers of the elements of $I$.

Definition 2.3. An $A\left[F^{e}\right]$-module is an $A$-module $M$ together with an $A$-linear map

$$
\mathcal{V}_{M}^{e}: F_{A}^{e *} M \rightarrow M
$$

A morphism between two $A\left[F^{e}\right]$-modules $\left(M, \mathcal{V}_{M}^{e}\right)$ and $\left(N, \mathcal{V}_{N}^{e}\right)$ is an $A$-linear map $\varphi: M \rightarrow N$ such that the following diagram commutes:

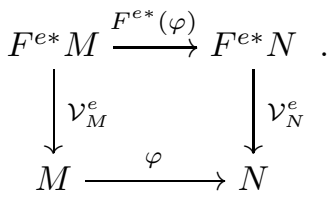

In fact, we can consider $F^{e} M$ as a $p^{e}$-linear map from $M \rightarrow M$; as such it is not $A$-linear but we have $F^{e}(a m)=a^{p^{e}} F^{e}(m), a \in A^{e}, m \in M$. Furthermore, an $A\left[F^{e}\right]$-module $\left(M, \mathcal{V}_{M}^{e}\right)$ is called a unite $A\left[F^{e}\right]$-module if $\mathcal{V}_{M}^{e}$ is an isomorphism (cf. [1. page 17, Definition 2.6]).

Remark 2.4. Since $F^{e *} A$ is canonically isomorphic to $A$, then $A$ is a unite $A\left[F^{e}\right]$ module, but an ideal $I \subset A$ is not a unite $A\left[F^{e}\right]$-module in general, as the inclusion $I^{\left[p^{e}\right]} \subseteq I$ can be strict. For a multiplicatively closed subset $S$ of $A$, the structural $\operatorname{map} \mathcal{V}_{S^{-1} A}^{e}: A^{e} \otimes S^{-1} A \rightarrow S^{-1} A$ is an isomorphism (see [1]).

The following definition is introduced in [12]:

Definition 2.5. Let $\left(M, \mathcal{V}^{e}\right)$ be an $A\left[F^{e}\right]$-module. We define $G(M)$ as the inverse limit generated by the structural map $\mathcal{V}^{e}$, i.e.

$$
G(M):=\lim _{\longleftarrow}\left(\cdots \rightarrow F^{3 e *} M \stackrel{F^{2 e *} \mathcal{V}^{e}}{\longrightarrow} F^{2 e *} M \stackrel{F^{e *} \mathcal{V}^{e}}{\longrightarrow} F^{e *} M \stackrel{\mathcal{V}^{e}}{\longrightarrow} M\right) .
$$

Note that there are natural maps $\pi_{e}: G(M) \rightarrow F^{e *} M$. Moreover, the maps $F^{e *} \pi_{r}: F^{e *} G(M) \rightarrow F^{e(r+1) *} M$ are compatible with the maps defining $G(M)$ and thus by the universal property of inverse limits, the map $F^{{ }^{*}} G(M) \rightarrow G(M)$ defines the natural $A\left[F^{e}\right]$-module structure on $G(M)$.

Proposition 2.6. (12, Proposition 1.2] and [1, Proposition 4.1]) Let $A$ be regular and $F$-finite and $M$ an $A\left[F^{e}\right]$-module. Then $G(M)$ is a unite $A\left[F^{e}\right]$-module. 
In order to extend the Matlis duality functor $D(-)=\operatorname{Hom}\left(-, E_{A}\right)$ where, $E_{A}$ is the injective hull of the residue field, the functor $\mathcal{D}$ from $A\left[F^{e}\right]$-modules to $A\left[F^{e}\right]$ modules is defined as follows ( [1, Section 4]):

Let $\left(M, \mathcal{V}^{e}\right)$ be an $A\left[F^{e}\right]$-module. We define

$$
\mathcal{D}(M)=\lim _{\longrightarrow}\left(D(M) \stackrel{D\left(\mathcal{V}^{e}\right)}{\longrightarrow} D\left(F^{e *} M\right) \stackrel{D\left(F^{e *}(\mathcal{V})\right.}{\longrightarrow} D\left(F^{2 e *} M\right) \longrightarrow \ldots\right) .
$$

An element $m \in M$ is called $F$-nilpotent if $F^{r e}(m)=0$ for some $r$. Then $M$ is called $F$-nilpotent if $F^{e r}(M)=0$ for some $r \geq 0$. It is possible that every element of $M$ is $F$-nilpotent but $M$ itself is not.

Below, we recall some properties of the functor $\mathcal{D}$.

Proposition 2.7. Let $A$ be a complete regular local ring.

(a) On the subcategory of $A\left[F^{e}\right]$-modules which are cofinite (i.e. satisfy the descending chain condition for submodules) as A-modules, the functor $\mathcal{D}$ is exact and $\mathcal{D}(N)$ is a finitely generated unite $A\left[F^{e}\right]$-module for every cofinite module $N$ (cf. [19, Theorem 4.2(i)] and [1, Proposition 4.16]).

(b) Let $M$ be an $A\left[F^{e}\right]$-module that is finitely generated or cofinite as an $A$ module. Then $\mathcal{D}(\mathcal{D}(M)) \cong G(M)$ (cf. [1, Proposition 4.17]).

(c) Let $M$ be an $A\left[F^{e}\right]$-module which is a cofinite A-module. Then $M$ is Fnilpotent if and only if $\mathcal{D}(M)=0$ (cf. [19, Theorem 4.2(ii)] and [1, Proposition 4.20]).

Let $A$ be a regular local ring and let $I$ be an ideal of $A$. As we have seen (following [1, page 19]) that $A$ is an $A\left[F^{e}\right]$-module and this structure comes down to its localization. The local cohomology modules $H_{I}^{i}(A)$ of $A$ with support in $I$ can be calculated as the cohomology modules of the Čech complex

$$
\check{C}\left(A ; x_{1}, \ldots, x_{n}\right)=A \rightarrow A_{x_{i}} \rightarrow A_{x_{i} x_{j}} \rightarrow A_{x_{1} x_{2} \cdots x_{n}}
$$

where, $I$ is generated by $x_{1}, x_{2}, \ldots, x_{n}$. As the category of unite $A\left[F^{e}\right]$-modules is an abelian subcategory of the category of $A\left[F^{e}\right]$-modules, the modules $H_{I}^{i}(A)$ are unite $A\left[F^{e}\right]$-modules for all $i \in \mathbb{Z}$. For formal local cohomology modules, the situation is a bit more complicated, however, we show that these kind of modules have unite $A\left[F^{e}\right]$-modules structure, where $A$ is $F$-finite.

Theorem 2.8. Let $(A, \mathfrak{m})$ be a regular $F$-finite local ring. Then

$$
G\left(H_{\mathfrak{m}}^{i}(A / I)\right) \cong \lim _{\longleftarrow} H_{\mathfrak{m}}^{i}\left(A / I^{n}\right), i \in \mathbb{Z}
$$

which is a unite $A\left[F^{e}\right]$-module. In particular,

$$
\lim _{n} H_{\mathfrak{m}}^{i}\left(A / I^{n}\right) \cong H_{\mathfrak{m}}^{i}\left(\hat{A}^{I}\right), i \in \mathbb{Z}
$$

as $A\left[F^{e}\right]$-modules, where $\hat{A}^{I}$ is the completion of $A$ along $I$. 
Proof. By what we have seen above, $H_{\mathfrak{m}}^{i}(A / I)$ is an $A\left[F^{e}\right]$-module for all $i \in \mathbb{N}$. Now, we may apply functor $G(-)$ to $H_{\mathfrak{m}}^{i}(A / I)$ :

$$
G\left(H_{\mathfrak{m}}^{i}(A / I)\right)=\lim _{\longleftarrow}\left(\cdots \stackrel{F^{2 e *} \mathcal{V}^{e}}{\longrightarrow} H_{\mathfrak{m}}^{i}\left(A / I^{\left[p^{2 e}\right]}\right) \stackrel{F^{e *} \mathcal{V}^{e}}{\longrightarrow} H_{\mathfrak{m}}^{i}\left(A / I^{\left[p^{e}\right]}\right) \stackrel{\mathcal{V}^{e}}{\longrightarrow} H_{\mathfrak{m}}^{i}(A / I)\right) .
$$

By virtue of Proposition 2.6 $G\left(H_{\mathfrak{m}}^{i}(A / I)\right)$ is a unite $A\left[F^{e}\right]$-module. Notice that, the right hand side is nothing but $\lim _{e} H_{\mathfrak{m}}^{i}\left(A / I^{\left[p^{e}\right]}\right)$.

On the other hand, one has $\lim _{n} H_{\mathfrak{m}}^{i}\left(A / I^{n}\right) \cong \operatorname{Hom}_{A}\left(H_{I}^{\operatorname{dim} A-i}(A), E\right)$, where $E:=E(A / \mathfrak{m})$ is the injective hull of the residue field. The natural map

$$
F^{e *} \operatorname{Hom}_{A}\left(H_{I}^{\operatorname{dim} A-i}(A), E\right) \rightarrow \operatorname{Hom}_{A}\left(H_{I}^{\operatorname{dim} A-i}(A), E\right)
$$

by sending $r \otimes \varphi$ to $r F^{e *}(\varphi)$ is an isomorphism of $A\left[F^{e}\right]$-modules $(r \in A$ and $\varphi \in$ $\left.\operatorname{Hom}_{A}\left(H_{I}^{\operatorname{dim} A-i}(A), E\right)\right)$. To this end note that $H_{I}^{\operatorname{dim} A-i}(A)$ and $E \cong H_{\mathfrak{m}}^{\operatorname{dim} A}(A)$ carry natural unite $A\left[F^{e}\right]$-structure. Thus, $\varliminf_{n} H_{\mathfrak{m}}^{i}\left(A / I^{n}\right)$ is a unite $A\left[F^{e}\right]$-module for each $i \in \mathbb{Z}$.

In order to complete the proof, it is enough to show that $\lim _{e} H_{\mathfrak{m}}^{i}\left(A / I^{\left[p^{e}\right]}\right) \cong$ $\lim _{n} H_{\mathfrak{m}}^{i}\left(A / I^{n}\right)$. For this reason, consider the decreasing family of ideals $\left\{I^{\left[p^{e}\right]}\right\}_{e}$. Clearly, its topology is equivalent to the $I$-adic topology on $A$. Thus, by [26, Lemma 3.8] there exists a natural isomorphism

$$
\lim _{e} H_{\mathfrak{m}}^{i}\left(A / I^{\left[p^{e}\right]}\right) \cong \lim _{\longleftarrow} H_{\mathfrak{m}}^{i}\left(A / I^{n}\right)
$$

for all $i \in \mathbb{Z}$.

The last part follows by [12, Proposition 2.1].

\section{Frobenius DePth}

Let $A$ be a regular local $F$-finite ring of characteristic $p>0$ and let $I$ be an ideal of $A$. As we have seen in the previous section the formal local cohomology modules, are unite $A\left[F^{e}\right]$-modules. In the present section we use the unite $A\left[F^{e}\right]$ structure of $\lim _{n} H_{\mathfrak{m}}^{i}\left(A / I^{n}\right)$ in order to prove our results.

Proposition 3.1. Let $(A, \mathfrak{m})$ be a regular local and $F$-finite ring. Then $\lim _{n} H_{\mathfrak{m}}^{i}\left(A / I^{n}\right)=$ 0 if and only if $H_{\mathfrak{m}}^{i}(A / I)$ is F-nilpotent.

Proof. By the assumptions $A$ is $F$-finite that is $A^{e}$ is a finitely generated $A$-module. Then tensoring with $A^{e}$ commutes with the inverse limit, as $A^{e}$ is a free right $A$ module (cf. [12, Proposition 1.1(b)]). Thus, we have

$$
A^{e} \otimes_{A} \widehat{A}=A^{e} \otimes_{A}\left(\lim _{n} A / I^{n}\right) \cong \lim _{\longleftarrow} A^{e} / A^{e} I^{n}=\lim _{n} A^{e} / I^{n\left[p^{e}\right]} A^{e} \cong \widehat{A^{e}} .
$$

On the other hand, since the Frobenius action is the same in both $H_{\mathfrak{m}}^{i}(A / I)$ and $H_{\widehat{\mathfrak{m}}}^{i}(\widehat{A} / I \widehat{A})$, so we may assume that $A$ is a complete regular local $F$-finite ring. 
As $H_{\mathfrak{m}}^{i}(A / I)$ is an $A\left[F^{e}\right]$-module which is a cofinite $A$-module, then $\mathcal{D}\left(H_{\mathfrak{m}}^{i}(A / I)\right)$ is a finitely generated unite $A\left[F^{e}\right]$-module (cf. 2.7(a)) and therefore

$$
\mathcal{D}\left(\mathcal{D}\left(H_{\mathfrak{m}}^{i}(A / I)\right)\right) \cong D\left(\mathcal{D}\left(H_{\mathfrak{m}}^{i}(A / I)\right)\right) .
$$

As the functor $D(-)$ transforms direct limits to inverse limits, then

$$
\begin{aligned}
D\left(\mathcal{D}\left(H_{\mathfrak{m}}^{i}(A / I)\right)\right) & =D\left(\lim _{\longrightarrow}\left(D\left(H_{\mathfrak{m}}^{i}(A / I)\right) \rightarrow D\left(F^{e *} H_{\mathfrak{m}}^{i}(A / I)\right) \rightarrow D\left(F^{2 e *} H_{\mathfrak{m}}^{i}(A / I)\right) \rightarrow \cdots\right)\right. \\
& \cong \lim _{\longleftarrow}\left(\cdots \rightarrow D\left(D\left(F^{2 e *} H_{\mathfrak{m}}^{i}(A / I)\right)\right) \rightarrow D\left(D\left(F^{e *} H_{\mathfrak{m}}^{i}(A / I)\right)\right) \rightarrow D\left(D\left(H_{\mathfrak{m}}^{i}(A / I)\right)\right)\right) \\
& \cong \lim _{e} H_{\mathfrak{m}}^{i}\left(A / I^{\left[p^{e}\right]}\right) .
\end{aligned}
$$

As we have seen in the proof of Theorem 2.8 $\lim _{e} H_{\mathfrak{m}}^{i}\left(A / I^{\left[p^{e}\right]}\right) \cong \lim _{n} H_{\mathfrak{m}}^{i}\left(A / I^{n}\right)$. Therefore, $H_{\mathfrak{m}}^{i}(A / I)$ is $F$-nilpotent if and only if $\mathcal{D}\left(H_{\mathfrak{m}}^{i}(A / I)\right)=0$ (2.7(c)) if and only if $\lim _{n} H_{\mathfrak{m}}^{i}\left(A / I^{n}\right)=0$.

Remark 3.2. Notice that in the Proposition 3.1 the $F$-finiteness of $A$ is vital, because it guarantees the $A\left[F^{e}\right]$ structure of the modules $\lim _{n} H_{\mathfrak{m}}^{i}\left(A / I^{n}\right)$. However, in the light of [1, Lemma 4.12] if $H_{I}^{\operatorname{dim} A-i}(A)$ is cofinite, then the module $\lim _{n} H_{\mathfrak{m}}^{i}\left(A / I^{n}\right)$ is $A\left[F^{e}\right]$-module.

Corollary 3.3. (21, Corollary 3.2]) Let $(A, \mathfrak{m})$ be a regular local ring and $I$ an ideal of $A$. Then $H_{I}^{\operatorname{dim} A-i}(A)=0$ if and only if $F^{e r}: H_{\mathfrak{m}}^{i}(A / I) \rightarrow H_{\mathfrak{m}}^{i}(A / I)$ is the zero map for some $r>0$.

Proof. As $\widehat{A}$ is a faithful flat $A$-module then by passing to the completion we may assume that $A$ is complete regular local ring. Let $H_{I}^{\operatorname{dim} A-i}(A)=0$, so it is cofinite. Then by the duality (1.1) in the introduction, one has $\lim _{n} H_{\mathfrak{m}}^{i}\left(A / I^{n}\right)=0$. Hence, Proposition 3.1 implies that $H_{\mathfrak{m}}^{i}(A / I)$ is $F$-nilpotent.

Conversely, assume that $H_{\mathfrak{m}}^{i}(A / I)$ is $F$-nilpotent. Then by Propostion 2.7(c) $\mathcal{D}\left(H_{\mathfrak{m}}^{i}(A / I)\right)=0$ and therefore one has $H_{I}^{\operatorname{dim} A-i}(A)=0$. To this end note that, $\mathcal{D}\left(H_{\mathfrak{m}}^{\operatorname{dim} A-i}\left(A / I^{\left[p^{e}\right]}\right)\right) \cong \operatorname{Ext}_{A}^{i}\left(A / I^{\left[p^{e}\right]}, A\right)$ for all $e \geq 0$ and the Frobenius powers of $I$ are cofinal with its ordinary powers.

In the light of Corollary 3.3. Lyubeznik 21] defined the F-depth of a local ring in order to give a solution to Grothendieck's Problem.

Definition 3.4. Let $(A, \mathfrak{m})$ be a local ring. The F-depth of $A$ is the smallest $i$ such that $F^{e r}$ does not send $H_{\mathfrak{m}}^{i}(A)$ to zero for any $r$.

One of elementary properties of F-depth shows that F-depth $A$ is equal to the F-depth of its $\mathfrak{m}$-adic completion, $\widehat{A}$ (cf. [21, Proposition 4.4]) because $H_{\mathfrak{m}}^{i}(A) \cong$ $H_{\mathfrak{\mathfrak { m }}}^{i}(\widehat{A})$. In the next result we give an alternative proof of [21, Lemma 4.2] to emphasize that F-depth of $A$ is bounded above by its Krull dimension. 
Proposition 3.5. Let $(A, \mathfrak{m})$ be a local ring and $I$ an ideal of $A$. Then $F^{e r}$ does not send $H_{\mathfrak{m}}^{\operatorname{dim} A}(A)$ to zero for any $r$. In particular, $0 \leq F \operatorname{depth} A \leq \operatorname{dim} A$.

Proof. Since the Frobenius action is the same in both $H_{\mathfrak{m}}^{i}(A)$ and $H_{\widehat{\mathfrak{m}}}^{i}(\widehat{A})$, so we may assume that $A$ is a complete local ring. Thus, by the Cohen's Structure Theorem $A \cong R / J$, where $R$ is a complete regular local ring and $J \subset R$ and ideal. In the contrary, assume that $H_{\mathfrak{m}}^{\operatorname{dim} A}(R / J)$ is $F$-nilpotent. Then, $\mathcal{D}\left(H_{\mathfrak{m}}^{\operatorname{dim} A}(R / J)\right)=0$ (cf. 2.7(c)) and with a similar argument given in the proof of Corollary 3.3, one can deduce the vanishing of $H_{J}^{0}(R)$. Hence, by virtue of (1.1), in the introduction, one has $\lim _{n} H_{\mathfrak{m}}^{\operatorname{dim}} A\left(R / J^{n}\right)=0$ which is a contradiction (cf. [26, Theorem 4.5]).

To investigate the other properties of $\mathrm{F}$-depth, in the next Theorem we compare the Frobenius depth of $A$ and $A^{\text {sh }}$. For this reason, let us recall some preliminaries. For a local ring $A$ we denote by $A^{\text {sh }}$ the strict Henselization of $A$. A local ring $(A, \mathfrak{m}, k)$ is said to be strictly Henselian if and only if every monic polynomial $f(T) \in A[T]$ for which $\overline{f(T)} \in k[T]$ is separable splits into linear factors in $A[T]$. For more advanced expositions on this topic we refer the interested reader to [23].

Proposition 3.6. Let $(A, \mathfrak{m})$ be a complete local ring. Then $\mathrm{F}$-depth $A=\mathrm{F}$-depth $A^{\text {sh }}$.

Proof. First assume that $A$ is a regular local ring. We show that F-depth $A / I=$ F-depth $(A / I)^{s h}$. Put F-depth $A / I=t$. Then, by virtue of Corollary 3.3 one has $H_{I}^{i}(A)=0$ for all $i>\operatorname{dim} A-t$. Due to the faithfully flatness of the inclusion $A \rightarrow A^{s h}$ and the fact that $A^{s h}$ is a regular local ring, it implies that $H_{I}^{i}\left(A^{s h}\right)=0$ for all $i>\operatorname{dim} A-t$. Again, using Corollary 3.3, it follows that F-depth $(A / I)^{s h} \geq t$. To this end note that $\operatorname{dim} A=\operatorname{dim} A^{s h}$ and $(A / I)^{s h}=A^{s h} / I A^{s h}$. With the similar argument one has F-depth $A / I \geq \mathrm{F}-\operatorname{depth}(A / I)^{s h}$. This completes the assertion.

Since $A$ is a complete local ring, then by virtue of Cohen's Structure Theorem, $A$ is a homomorphic image of a regular local ring $R$, i.e. $A=R / J$ for some ideal $J$ of $R$. Now, we are done by the previous paragraph. To this end note that

$$
\text { F-depth } A=\text { F-depth } R / J=\text { F-depth }(R / J)^{s h}=\text { F-depth } A^{s h} .
$$

Remark 3.7. From Proposition 3.6 and [21, Proposition 4.4] one may deduce that

$$
\text { F-depth } A=\text { F-depth }\left(\widehat{(\hat{A})^{s h}}\right),
$$

where, $A$ is a local ring.

In the following, we compare the invariants depth, F-depth and fgrade. Let us recall that the formal grade, fgrade $(I, R)$, is defined as the index of the minimal nonvanishing formal cohomology module, i.e., fgrade $(I, R)=\inf \left\{i \in \mathbb{Z} \mid \lim _{n} H_{\mathfrak{m}}^{i}\left(R / I^{n}\right) \neq\right.$ $0\}$. 
Theorem 3.8. Let $(A, \mathfrak{m})$ be a local $F$-finite ring and let $I$ be an ideal of $A$. Then

$$
\text { fgrade }(I, A) \leq \operatorname{depth} A \leq \text { F-depth } A \text {. }
$$

Proof. We have $\lim _{n} H_{\mathfrak{m} \hat{A}}^{i}\left(\hat{A} / I^{n} \hat{A}\right) \cong \lim _{n} H_{\mathfrak{m}}^{i}\left(A / I^{n} A\right)$ (cf. [26, Proposition 3.3]) and F-depth $A \cong$ F-depth $\hat{A}$ (cf. [21, Proposition 4.4]). As m-adic completion of an $F$-finite ring is again $F$-finite, Then, we may assume that $A$ is a complete local $F$-finite ring. Then $K=A / \mathfrak{m}$ is $F$-finite, then so is every finitely generated algebra over $K$.

By the Cohen's Structure Theorem there exists an $F$-finite regular local ring $(R, \mathfrak{n})$ with $A \cong R / J$ where, $J$ is an ideal of $R$.

Put fgrade $(J, R)=t$. Then by definition $\lim _{n} H_{\mathfrak{n}}^{i}\left(R / J^{n}\right)=0$ for all $i<t$. It follows from the Proposition 3.1 that $H_{\mathfrak{n}}^{i}(R / J)$ is $F$-nilpotent for all $i<t$, i.e. F-depth $R / J \geq t$. With a similar argument and again using Proposition 3.1 we have fgrade $(J, R) \geq \mathrm{F}$-depth $R / J$. Thus, $\operatorname{fgrade}(J, R)=\mathrm{F}$-depth $R / J$.

Now, we are done by [26, Lemma 4.8(b)], [6, Remark 3.1] and the previous paragraph. To this end note that

$$
\begin{aligned}
\operatorname{fgrade}(I, A) \leq \operatorname{depth} A & \leq \operatorname{fgrade}(J, R) \\
& =\mathrm{F}-\operatorname{depth} R / J \\
& =\mathrm{F}-\operatorname{depth} A .
\end{aligned}
$$

Let $A$ be a complete local ring containing a perfect field $k$ as its residue field, then $A$ satisfies the condition of Theorem 3.8, To this end, note that by Remark 2.1. $A$ is F-finite. Furthermore, by virtue of Cohen's Structure Theorem $A \cong R / J$ for some ideal $J \subset R$, where $R=k\left[\left[x_{1}, \ldots, x_{n}\right]\right]$ is a regular $F$-finite ring.

Corollary 3.9. Let $(A, \mathfrak{m})$ be a regular local and $F$-finite ring. Then we have

$$
\operatorname{depth} A / I \leq \operatorname{fgrade}(I, A)=\text { F-depth } A / I \leq \operatorname{dim} A / I .
$$

Proof. The assertion follows from what we have seen in the proof of Theorem 3.8 and [7, Remark 3.1].

Remark 3.10. (a) The necessary and sufficient conditions for small values of the F-depth of $A$ is given in [21, Corollary 4.6].

(1) F-depth $A>0$ if and only if $\operatorname{dim} A>0$.

(2) F-depth $A>1$ if and only if $\operatorname{dim} A \geq 2$ and the punctured spectrum of $A$ is formally geometrically connected.

Now, let $A$ be $F$-finite and $\operatorname{depth} A=0<\operatorname{dim} A$. Then, one has fgrade $(I, A)=$ $0<$ F-depth $A$. It shows that the inequality in Theorem 3.8 can be strict. 
(b) Keep the assumptions in Corollary 3.9. if F-depth $A / I>1$, then by [26, Lemma 5.4], one has $\operatorname{Supp}_{\hat{A}}(\hat{A} / I \hat{A}) \backslash\{\hat{\mathfrak{m}}\}$ is connected. To this end, note that $\hat{A}$ is a local ring so it is indecomposable.

\section{ENDOMORPHISM RINGS OF LOCAL COHOMOLOGY MODULES}

Let $R$ be a commutative algebra and $k \subset R$ be a field. We denote by $\operatorname{End}_{k}(R)$ the $k$-linear endomorphism ring. The ring of $k$-linear differential operators $\mathfrak{D}_{R \mid k} \subseteq$ $\operatorname{End}_{k}(R)$ generated by the $k$-linear derivations $R \rightarrow R$ and the multiplications by elements of $R$. By a $\mathfrak{D}_{R \mid k}$-module we always mean a left $\mathfrak{D}_{R \mid k}$-module. The injective ring homomorphism $R \rightarrow \mathfrak{D}_{R \mid k}$ that sends $r$ to the map $R \rightarrow R$ which is the multiplication by $r$, gives $\mathfrak{D}_{R \mid k}$ a structure of $R$-algebra. Every $\mathfrak{D}_{R \mid k}$-module $M$ is automatically an $R$-module via this map. The natural action of $\mathfrak{D}_{R \mid k}$ on $R$ makes $R$ a $\mathfrak{D}_{R \mid k}$-module. If $R=k\left[\left[x_{1}, \ldots, x_{n}\right]\right]$ is a formal power series ring of $n$ variables $x_{1}, \ldots, x_{n}$ over $k$, then $\mathfrak{D}_{R \mid k}$ is left and right Noetherian. Moreover, $\mathfrak{D}_{R \mid k}$ is a simple ring. Noteworthy, the local cohomology module $H_{I}^{i}(R), i \in \mathbb{Z}$ is a finitely generated $\mathfrak{D}_{R \mid k}$-module. For a more advanced exposition based on differential operators and undefined concepts the interested reader might consult 2. The similar results are true whenever $R=k\left[x_{1}, \ldots, x_{n}\right]$ is a polynomial ring of $n$ variables $x_{1}, \ldots, x_{n}$ over $k$ (cf. [5]). For a quick introduction in this topic we refer the reader to [20]. For brevity we often write $\mathfrak{D}_{R}$ for $\mathfrak{D}_{R \mid k}$ when there is no ambiguity about the field $k$.

Finally, we bring the following Lemma will be used later.

Lemma 4.1. Let $R$ be a commutative ring containing a field $k$. Let $M$ be both an $R$-module and $a \mathfrak{D}_{R}$-module. Then $\mathrm{Ann}_{\mathfrak{D}_{R}} M=0$ implies $\operatorname{Ann}_{R} M=0$.

Proof. Let $r \in \operatorname{Ann}_{R} M$ be an arbitrary element. As the endomorphism $\varphi_{r}: R \rightarrow R$ with $\varphi_{r}(s)=r s$ for all $s \in R$ is an element of $\mathfrak{D}_{R}$ so from $r s M=0$ (for all $s \in R$ ) we have $\varphi_{r}(s) M=0$. That is $\varphi_{r}(s) \in \operatorname{Ann}_{\mathfrak{D}_{R}} M=0$, i.e. $r s=\varphi_{r}(s)=0$, for all $s \in R$. Hence, we have $r=0$, as desired.

4.1. Characteristic 0 . Throughout this subsection $k$ denotes a field of characteristic zero. Let $R$ be either $k\left[\left|x_{1}, \ldots, x_{n}\right|\right]$, a formal power series ring or $k\left[x_{1}, \ldots, x_{n}\right]$ a polynomial ring of $n$ variables $x_{1}, \ldots, x_{n}$ over $k$. It is known that the local cohomology module $H_{I}^{i}(R), i \in \mathbb{Z}$ is a holonomic $\mathfrak{D}_{R}$-module [18, i.e. it is a finitely generated $\mathfrak{D}_{R}$-module with $d\left(H_{I}^{i}(R)\right)=n$. To this end note that $d\left(H_{I}^{i}(R)\right)$ is the so-called Bernstein dimension of $H_{I}^{i}(R)$ which is by the definition the Krull dimension of the characteristic variety $\operatorname{Char}\left(H_{I}^{i}(R)\right)$ (see [2] and [5]). In the following result we show that this is not the case for its endomorphism ring.

For the convenience of the reader let us recall two useful facts. 
Fact 4.2. (cf. 2] and [5])

(1) For any nonzero finitely generated $\mathfrak{D}_{R}$-module $M$, one has $n \leq d(M) \leq 2 n$.

(2) $d\left(\mathfrak{D}_{R}\right)=2 n$.

Proposition 4.3. Let $R$ be as above. Suppose that $H_{I}^{i}(R) \neq 0$, then $d\left(\operatorname{Hom}_{k}\left(H_{I}^{i}(R), H_{I}^{i}(R)\right)\right)=$ 2n. In particular $\operatorname{Hom}_{k}\left(H_{I}^{i}(R), H_{I}^{i}(R)\right)$ is not a holonomic $\mathfrak{D}_{R}$-module.

Proof. As $H_{I}^{i}(R)$ is a finitely generated $\mathfrak{D}_{R}$-module, then so is $\operatorname{Hom}_{k}\left(H_{I}^{i}(R), H_{I}^{i}(R)\right)$. As $H_{I}^{i}(R)$ is a non-zero $k$-vector space, it has a non-trivial endomorphism ring. Consider the homomorphism

$$
\mathfrak{D}_{R} \stackrel{f}{\longrightarrow} \operatorname{Hom}_{k}\left(H_{I}^{i}(R), H_{I}^{i}(R)\right)
$$

of $\mathfrak{D}_{R}$-modules defined by $f(P)(m)=P m, P \in \mathfrak{D}_{R}$ and $m \in H_{I}^{i}(R)$ for all $i \in \mathbb{Z}$. The homomorphism $f$ is injective, as $\mathfrak{D}_{R}$ is a simple ring. In the light of (4.1) and the fact that $d\left(\mathfrak{D}_{R}\right)=2 n$, the dimension of $\operatorname{Hom}_{k}\left(H_{I}^{i}(R), H_{I}^{i}(R)\right)$ is at least $2 n$. Hence, the assertion follows from Fact (4.2).

Theorem 4.4. Let $R$ be either $k\left[\left|x_{1}, \ldots, x_{n}\right|\right]$, a formal power series ring or $k\left[x_{1}, \ldots, x_{n}\right]$ a polynomial ring of $n$ variables $x_{1}, \ldots, x_{n}$ over $k$. Suppose that $H_{I}^{i}(R) \neq 0$. Then

$$
\operatorname{Ann}_{R}\left(\operatorname{Hom}_{R}\left(H_{I}^{i}(R), H_{I}^{i}(R)\right)\right)=0 .
$$

Proof. As $\operatorname{Hom}_{R}\left(H_{I}^{i}(R), H_{I}^{i}(R)\right)$ is a non zero $\mathfrak{D}_{R}$-module and $\mathfrak{D}_{R}$ is a simple ring one has $\operatorname{Ann}_{\mathfrak{D}_{R}}\left(\operatorname{Hom}_{R}\left(H_{I}^{i}(R), H_{I}^{i}(R)\right)\right)=0$. Now, we are done by Lemma 4.1 .

Now, we may recover a result of Lyubeznik.

Corollary 4.5. (cf. [18, Corollary 3.6]) Let $(R, \mathfrak{m})$ be a regular local ring containing a field of characteristic zero. Suppose that $H_{I}^{i}(R) \neq 0$. Then $\operatorname{Ann}_{R} H_{I}^{i}(R)=0$.

Proof. Let $\hat{R}$ be the $\mathfrak{m}$-adic completion of $R$. It is known that

$$
\operatorname{Ann}_{R}\left(H_{I}^{i}(R)\right)=\operatorname{Ann}_{\hat{R}}\left(H_{I \hat{R}}^{i}(\hat{R})\right)
$$

and $H_{I}^{i}(R) \otimes_{R} \hat{R} \cong H_{I \hat{R}}^{i}(\hat{R}) \neq 0$, because of the faithfully flatness of $\hat{R}$. Then, we may assume that $R$ is complete. Now we are done by virtue of Cohen's Theorem and Theorem 4.4. To this end, let $f \in \operatorname{Hom}_{R}\left(H_{I}^{i}(R), H_{I}^{i}(R)\right)$ be an arbitrary element and $r \in \operatorname{Ann}_{R}\left(H_{I}^{i}(R)\right)$ with $r z=0$, for every $z \in H_{I}^{i}(R)$. Hence, for every $z \in H_{I}^{i}(R)$ one has $r f(z)=f(r z)=0$ so $\operatorname{Ann}_{R}\left(H_{I}^{i}(R)\right) \subseteq$ $\operatorname{Ann}_{R}\left(\operatorname{Hom}_{R}\left(H_{I}^{i}(R), H_{I}^{i}(R)\right)\right)$. 
4.2. characteristic $p$. Throughout this subsection $R$ is a commutative Noetherian ring containing a field of positive characteristic.

Proposition 4.6. Let $(R, \mathfrak{m})$ be a F-finite regular local ring. Then $\operatorname{Hom}_{R}\left(H_{I}^{i}(R), H_{I}^{i}(R)\right)$ is a unite $R\left[F^{e}\right]$-module.

Proof. As mentioned before (page 5), for a given $i, H_{I}^{i}(R)$ is a unite $R\left[F^{e}\right]$-module. Now we are done by [1, Corollary 4.10].

Lemma 4.7. Let $M$ be an Artinian $R$-module ( $R$ is an arbitrary ring with no restriction on its characteristic). Then there exists an isomorphism

$$
\Phi: \operatorname{Hom}_{R}(M, M) \rightarrow \operatorname{Hom}_{\widehat{R}}(D(M), D(M)),
$$

which sends every $\varphi \in \operatorname{Hom}_{R}(M, M)$ to $\Phi(\varphi): \theta \mapsto \theta \circ \varphi$, where $\theta \in D(M)$.

Whenever $R$ is complete and $M$ is a finitely generated $R$-module, we have again such isomorphism.

Proof. We prove for the case $M$ is an Artinian module. For a finite module over a complete local ring the argument is the same. It is clear that the homomorphism $\Phi$ is well-defined. It is enough to show that it is injective and onto.

( $\Phi$ is injective) Let $0 \neq \varphi$ so there exists a nonzero elemnet $x \in M$ such that $\varphi(x) \neq 0$ in $M$. The exact sequence $0 \rightarrow\langle\varphi(x)\rangle \rightarrow M \rightarrow(M /\langle\varphi(x)\rangle):=L \rightarrow 0$ implies the following short exact sequence $0 \rightarrow D(L) \rightarrow D(M) \rightarrow D(\langle\varphi(x)\rangle) \rightarrow 0$. It is clear that $D(\langle\varphi(x)\rangle) \neq 0$, otherwise $D(L) \cong D(M)$. Using Matlis duality it implies that $L \cong M$ so $\varphi(x)=0$, that is contradiction. Thus, there exists a nonzero $\operatorname{map} \theta:\langle\varphi(x)\rangle \rightarrow E(R / \mathfrak{m})$. We may extend it to the nonzero map $\theta: M \rightarrow E(R / \mathfrak{m})$ so $\theta \circ \varphi \neq 0$, as required.

( $\Phi$ is onto) Let $\psi \in \operatorname{Hom}_{\widehat{R}}(D(M), D(M))$. We define $\psi$ to send $\theta \in D(M)$ to $\theta \circ D(\psi)$, where $D(\psi): D(D(M)) \rightarrow D(D(M))$. As $M$ is Artinian, $D(D(M)) \cong M$ so $D(\psi): M \rightarrow M$. Then $\Phi(D(\psi))=\theta \circ D(\psi)=\psi_{\theta}$. Thus, $\Phi(D(\psi))=\psi$, i.e. $\Phi$ is onto.

In the following, the endomorphism ring $\operatorname{Hom}_{R}\left(H_{I}^{i}(R), H_{I}^{i}(R)\right)$ is a unite $R\left[F^{e}\right]$ module without any need for $R$ to be $F$-finite.

Proposition 4.8. Let $(R, \mathfrak{m})$ be a regular local ring. Suppose that $H_{I}^{i}(R)$ is an Artinian $R$-module. Then $\operatorname{Hom}_{R}\left(H_{I}^{i}(R), H_{I}^{i}(R)\right)$ is a unite $R\left[F^{e}\right]$-module.

Proof. By virtue of Lemma 4.7 and the fact that $D\left(H_{I}^{i}(R)\right)$ is finitely generated one has

$$
F^{e}\left(\operatorname{Hom}_{R}\left(H_{I}^{i}(R), H_{I}^{i}(R)\right)\right) \cong \operatorname{Hom}_{R}\left(F^{e}\left(D\left(H_{I}^{i}(R)\right)\right), F^{e}\left(D\left(H_{I}^{i}(R)\right)\right)\right.
$$


It follows from [1, Lemma 4.12] that

$$
\operatorname{Hom}_{R}\left(F^{e}\left(D\left(H_{I}^{i}(R)\right)\right), F^{e}\left(D\left(H_{I}^{i}(R)\right)\right) \cong \operatorname{Hom}_{R}\left(D\left(F^{e}\left(H_{I}^{i}(R)\right)\right), D\left(F^{e}\left(H_{I}^{i}(R)\right)\right) .\right.\right.
$$

As $H_{I}^{i}(R)$ is a unite $R\left[F^{e}\right]$-module, then

$$
\operatorname{Hom}_{R}\left(D\left(F^{e}\left(H_{I}^{i}(R)\right)\right), D\left(F^{e}\left(H_{I}^{i}(R)\right)\right) \cong \operatorname{Hom}_{R}\left(D\left(H_{I}^{i}(R)\right), D\left(H_{I}^{i}(R)\right)\right) .\right.
$$

Once again, using Lemma 4.7 the proof is complete.

Now we are ready to prove the main result of this subsection but before it we need some preliminaries. It is noteworthy to say that unite $R\left[F^{e}\right]$-modules have the structure of $\mathfrak{D}$-modules in the case of characteristic $p>0$ (cf. [19] and [1]).

Definition 4.9. A reduced $F$-finite ring is said to be strongly $F$-regular, if for all $c \in R$ not in any minimal prime, there exists $q=p^{e}$ such that the map $R \rightarrow R^{1 / q}$ sending $1 \mapsto c^{1 / q}$ splits as an $R$-module homomorphism. Here, $R^{1 / q}$ is the over-ring of $q$ th roots of elements in $R$.

The strongly $F$-regular rings were introduced by Hochster and Huneke in [15]. It is known that a regular, $F$-finite ring is strongly $F$-regular.

Definition 4.10. An $F$-finite ring $\mathrm{R}$ has finite $\mathrm{F}$-representation type if there exists a finite set $\mathcal{S}$ of isomorphism classes of $R$ - modules such that any indecomposable $R$-module summand of $R^{1 / q}$, for any $q=p^{e}$, is isomorphic to some element of $\mathcal{S}$.

Note that an $F$-finite regular ring has finite $F$-representation type, see [29] for more information.

Theorem 4.11. Let $(R, \mathfrak{m})$ be a complete ring. If $R$ is strongly $F$-regular and has finite F-representation type. Suppose that $\operatorname{Hom}_{R}\left(H_{I}^{i}(R), H_{I}^{i}(R)\right)$ is a unite $R\left[F^{e}\right]$ module. Then $\operatorname{Ann}_{R}\left(\operatorname{Hom}_{R}\left(H_{I}^{i}(R), H_{I}^{i}(R)\right)\right)=0$.

Proof. As $\operatorname{Hom}_{R}\left(H_{I}^{i}(R), H_{I}^{i}(R)\right)$ is a unite $R\left[F^{e}\right]$-module, it then follows from [1, Proposition 3.6] that $\operatorname{Hom}_{R}\left(H_{I}^{i}(R), H_{I}^{i}(R)\right)$ has a $\mathfrak{D}_{R}$-module structure. On the other hand by [29, Theorem 4.2.1], $\mathfrak{D}_{R}$ is a simple ring. Then one has

$$
\operatorname{Ann}_{\mathfrak{D}_{R}} \operatorname{Hom}_{R}\left(H_{I}^{i}(R), H_{I}^{i}(R)\right)=0 .
$$

Now we are done by Lemma 4.1 ,

As a consequence of Theorem 4.11 we can recover a result of Huneke-Koh.

Corollary 4.12. (cf. [16, Lemma 2.2]) Let $(R, \mathfrak{m})$ be a regular local ring containing a field $k$ of characteristic $p>0$. If $H_{I}^{i}(R) \neq 0$, then $\operatorname{Ann}_{R} H_{I}^{i}(R)=0$. 
Proof. Once again similar to what we have seen in the proof of Theorem 4.5 we may assume that $R$ is a complete local ring. Without loss of generality, we may assume that $k=R / \mathfrak{m}$. Using a suitable gonflement of $R$ there exists a regular local ring $(S, \mathfrak{n})$ such that $S / \mathfrak{n}$ is the algebraic closure of $k$ and $(S, \mathfrak{n})$ is faithful flat over $(R, \mathfrak{m})$, see [4, Chapter IX, Appendice 2]. By faithful flatness of $S$ one has $S / \mathfrak{m} S=S / \mathfrak{n}$. By passing over $S$ we may assume that $k$ is an algebraically closed field. Therefore, $k$ is a perfect field. In the light of Remark 2.1 we observe that $R$ is $F$-finite. Now the claim follows from the Proposition 4.6 and Theorem 4.11.

Acknowledgements. I am deeply grateful to the referee for his/her careful reading of the manuscript, appropriate and constructive suggestions to improve the paper. This project is based on a question asked by Professor Josep Àlvarez-Montaner on the comparison of F-depth and fgrade when the author was visiting the Universitat Politècnica de Catalunya, in September 2013. Hereby, I would like to express my thanks to the Universitat Politècnica and specially, to Josep, for their support and warm hospitality. I am also strongly indebted with Josep for several fruitful discussions. I would like to mentioned that when the author was finishing this project, he started a joint project with Alberto F. Boix, and gave a characteristic free proof of the result of Huneke-Koh and Lyubeznik on the annihilator of local cohomology modules.

\section{REFERENCES}

[1] M. Blickle, The Intersection Homology D-Module in Finite Characteristic., Ph.D. thesis, University of Michigan (2001).

[2] J. E. Björk, Rings of differential operators. Amsterdam North-Holland (1979).

[3] M. Brodmann and R.Y. Sharp, Local cohomology: an algebraic introduction with geometric applications. Cambridge Univ. Press, 60, Cambridge, (1998).

[4] N. Bourbaki , Éléments de mathématique.Algébre commutative Chapters 8 et 9, Springer, (2008).

[5] S. C. Coutinho, A primer of algebraic D-modules, London Mathematical Society Student Texts, Cambridge University Press, (1995).

[6] M. Eghbali, On Artinianness of formal local cohomology, colocalization and coassociated primes., Math. Scand. 113, 5-19 (2013).

[7] M. Eghbali, On set theoretically and cohomologically complete intersection ideals., Canad. Math. Bull. 57(3), 477-484 (2014).

[8] M. Eghbali and P. Schenzel, On an Endomorphism Ring of Local Cohomology., Comm. in Alg. 40, 4295-4305 (2012).

[9] A. Grothendieck, Local cohomology. Lecture notes in Mathematics, 41, Springer, Heidelberg (1967).

[10] A. Grothendieck, Cohomologie locale des faisceaux et théorèmes de Lefschetz locaux et globaux (SGA 2). North-Holland (1968). 
[11] R. Hartshorne, Cohomological dimension of algebraic varieties. Ann. of Math. 88(2), 403-450 (1968).

[12] R. Hartshorne and R. Speiser, Local cohomological dimension in characteristic p. Ann. of Math. 105(2), 45-79 (1977).

[13] M. Hellus and J. Stückrad, On endomorphism rings of local cohomology modules. Proc. Amer. Math. Soc. 136(7), 2333-2341 (2008).

[14] M. Hochster and C. Huneke, Indecomposable canonical modules and connectedness., In: Heinzer, W., Huneke, C., Sally, J., eds. Commutative Algebra: Syzygies, Multiplicities, and Birational Algebra. Contemporary Math. Vol. 159, pp. 97-208 (1994).

[15] M. Hochster and C. Huneke, Tight closure and strong F-regularity., Mém. Soc. Math. France (N.S.) 38, 119-133 (1989).

[16] C. Huneke and J. Koh, cofiniteness and vanishing of local cohomology modules., Math. Proc. Cambridge Philoos. Soc. 1103, 421-429 (1991).

[17] E. Kunz, Characterization of regular local rings in characteristic p. Amer J. Math. 91, 772784 (1969).

[18] G. Lyubeznik, Finiteness properties of local cohomology modules (an application of Dmodules to commutative algebra, Invent. Math. 1131, 41-55 (1993).

[19] G. Lyubeznik, F-modules: an application to local cohomology and D-modules in characteristic $p>0$. Journal für reine und angewandte Mathematik 491, 65-130 (1997).

[20] G. Lyubeznik, Injective dimension of D-modules: a characteristic-free approach. J. Pure and Appl. Algebra 149 205-212 (2000).

[21] G. Lyubeznik, On the vanishing of local cohomology in characteristic $p>0$. Composito Math. 142, 207-221 (2006).

[22] W. Mahmood and P. Schenzel, On invariants and endomorphism rings of certain local cohomology modules. J. Algebra, 372, 56-67 (2012).

[23] J. S. Milne, Étale cohomology. Princeton Mathematical Series 33, Princeton University Press (1980).

[24] A. Ogus, Local cohomological dimension of Algebraic Varieties. Ann. of Math. 98(2), 327365 (1973).

[25] C. Peskine and L. Szpiro, Dimension projective finie et cohomologie locale. Publ. Math. I.H.E.S., 42, (1973), 323-395.

[26] P. Schenzel, On formal local cohomology and connectedness. J. Algebra, 315(2), 894-923 (2007).

[27] P. Schenzel, On endomorphism rings and dimensions of local cohomology modules. Proc. Amer. Math. Soc. 137, 1315-1322 (2009).

[28] P. Schenzel, Matlis duals of local cohomology modules and their endomorphism rings. Archiv Math. 95, 115-123 (2010).

[29] K. E. Smith and M. Van den Bergh, Simplicity of rings of differential operators in prime characteristic., Proc. London Math. Soc., 75(3), 32-62 (1997).

School of Mathematics, Institute for Research in Fundamental Sciences (IPM), P.

O. Box: 19395-5746, Tehran-Iran.

E-mail address: m.eghbali@yahoo.com 